\title{
Surface Blemish Detection from Passive Imagery Using Learned Fuzzy Set Concepts
}

\author{
Sabri Gurbuz, Albrecht Carver and Robert Schalkoff \\ Department of Electrical and Computer Engineering \\ Clemson University \\ Clemson, SC 29634-0915 \\ CONF-9704176-- \\ Phone: 864-656-5913 FAX: 864-656-7220 \\ e-mail: rjschal@clemson.edu
}

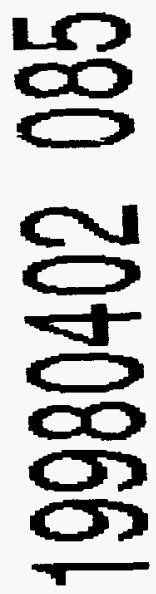

\begin{abstract}
-
An image analysis method for real-time surface blemish detection using passive imagery and fuzzy set concepts is described. The method develops an internal knowledge representation for surface blemish characteristics on the basis of "experience", thus facilitating autonomous learning based upon positive and negative exemplars. The method incorporates fuzzy set concepts in the learning subsytem and image segmentation algorithms, thereby mimicking human visual perception. This enables a generic solution for color image segmentation.
\end{abstract}

\section{INTRODUCTION}

The human visual system uses color and texture as significant cues to discriminate regions in images. This has led the development of machine vision systems relying principally on two approaches for image segmentation: color space analysis and texture analysis. This paper presents a novel color-based analysis technique for classifying image regions using fuzzy set concepts.

Why use a fuzzy approach for color image analysis? The answer lies in that fuzzy concepts embody non-linear domain knowledge, just like human visual perception. How does the human visual system (HVS) perceive the colors in photographic imagery? To answer this, consider a pho-

This work was funded by the U. S. Department of Energy Morgantown Energy Technology Center, Contract METC DE-AC21-92MC29115. tographic image which depicts a surface painted black with a yellow sticker adhering to it and a rusty patch showing through the paint. If a human is shown a single yellow pixel and asked "Does this pixel belong to the yellow sticker or to the rust patch?", the HVS might classify the pixel color using its internal knowledge representativi. " yellow stickers and rust. Some measure of confidence of its being a member of the yellow sticker and its being a member of the rust patch might then be established. The knowledge representation the HVS uses for these assignments is developed from past "experiences", or the lack thereof. The HVS then compares these two measures and assigns a linguistic meaning to the pixel, such as "it belongs to the yellow sticker." If this linguistic answer is an absolute assignment, this answer is referred to as "crisp." If, on the other hand, the HVS is unable to confidently assign the pixel, the answer is referred to as "uncertain."

By analogy with the HVS approach. our fuzzy approach to image analysis consists of three phases: the learning phase, in which the system gains "experience" and converts it into a knowledge representation: the segmentation phase in which confidence measures are assigned using this knowledge; and the defuzzification phase in which the linguistic assignment is made. The knowledge representations in this approach are mappings from pixel colors and locations to con-

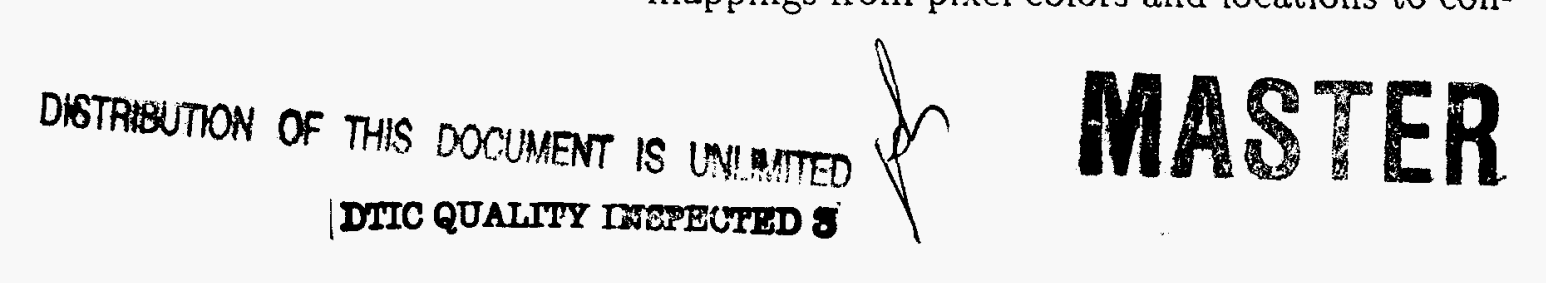




\section{DISCLAIMER}

This report was prepared as an account of work sponsored by an agency of the

United States Government. Neither the United States Government nor any agency thereof, nor any of their employees, makes any warranty, express or implied, or assumes any legal liability or responsibility for the accuracy, completeness, or usefulness of any information, apparatus, product, or process disclosed, or represents that its use would not infringe privately owned rights. Reference herein to any specific commercial product, process, or service by trade name, trademark, manufacturer, or otherwise does not necessarily constitute or imply its endorsement, recommendation, or favoring by the United States Government or any agency thereof. The views and opinions of authors expressed herein do not necessarily state or reflect those of the United States Government or any agency thereof. 
fidences, and are referred to as fuzzy membership functions. The algorithms are presented for each of three phases, learning, segmentation, and defuzzification, in Sections II, III, and IV, respectively. In addition, an algorithm application involving visual inspection is shown. Mixed waste drums are imaged and an autonomous decision is made as to whether the drums are sufficiently rusty and therefore likely to leak. Section V describes this application and presents results. Concluding remarks are then presented in Section VI.

\section{TRAINING: THE LEARNING PHASE}

The primary steps in training are:

1. Create training data sets formed from pixels indicated by human trainer as positive and negative exemplars;

2. Cluster each of the data sets (one positive and one negative) using the c-means algorithm, and

3. Construct fuzzy membership functions for each cluster using histograms of hue, intensity, and saturation values.

Training consists of positive and negative training where both positive and negative exemplars are introduced to the system. After inputting the positive exemplars, the system computes a user-definable number of clusters, up to eight, using the c-means algorithm for each hue, saturation, and intensity component of the exemplars [6], [4]. Clusters with less than $T$ (some threshold value) members are discarded because of the possibility of being noise pixels or background pixels in the exemplar regions. The remaining data are then reclustered. Following this, the system computes color space statistics of each cluster, which define a confidence function or membership function of the cluster, based on the histogram properties [1].

The next step is to segment the image using these positive cluster similarity membership functions in order to generate misclassified (false- positive) or "negative" pixels. In the same way, negative pixels are clustered using the c-means algorithm. Then, the histogram of each cluster is calculated to produce negative cluster similarity membership functions (SMF), and all SMFs are stored in a database.

SMFs are constructed as follows. Let $P_{j}$ and $N_{j}$, be positive cluster and negative clusters, respectively, where $j=1,2, \ldots, \mathrm{k}$ ( $\mathrm{k}$ is the number of clusters produced by the c-means algorithm for an exemplar data set). Each cluster similarity membership function is given by

$$
\begin{aligned}
\mu_{P_{j}}(i) & =\frac{\operatorname{histogram}\left[P_{j}(i)\right]}{\max \left[\operatorname{histogram}\left[P_{j}(i)\right]\right]} . \\
\mu_{N_{j}}(i) & =\frac{\text { histogram }\left[N_{j}(i)\right]}{\max \left[\operatorname{histogram}\left[N_{j}(i)\right]\right]},
\end{aligned}
$$

where histogram represents the relative frequency of occurrence of the various pixel values (gray levels) in the cluster [4], and $i$ represents the pixel value of the input, i.e., $i \in[0,255]$.

\section{SEGMENTATION: RECOGNITION PHASE}

After acquiring the RGB (red, green, and blue) image, it is converted to an HSI (hue, saturation, and intensity) representation in order to make color and intensity independent of one another [2], [3]. The block diagram of segmentation algorithm is shown in Figure 1. The procedure for the image segmentation process can be summarized as follows:

1. Fuzzify the $\mathrm{H}, \mathrm{S}$. and I planes using positive membership functions:

2. Fuzzify the $\mathrm{H}, \mathrm{S}$, and I planes using negative membership functions;

3. Accumulate fuzzified image of positive and negative membership functions separately using fuzzy union;

4. Construct neigborhood support for negative fuzzy set using similarity measure, and

5. Defuzzify using inference operation to generate segmented image. 


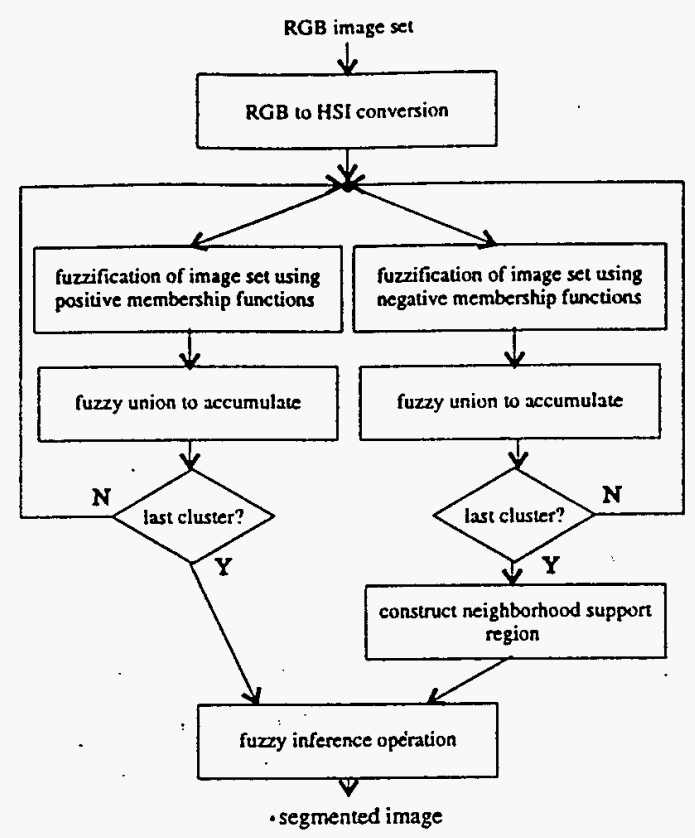

Fig. 1. Block diagram of segmentation algorithm

Figure 2 shows the block diagram of the fuzzification algorithm. In the fuzzification process using the positive cluster set, the pixels in each of the hue, saturation, and intensity planes of the input image are analyzed and assigned membership values in the fuzzy sets $H_{j}^{+}$, $S_{j}^{+}$, and $I_{j}^{+}$, respectively, where $j$ indicates the $j^{\text {th }}$ positive cluster. This is done by matching the pixels' color properties with those of the cluster. The pixels of the HSI image are then assigned a membership value in fuzzy set $P_{j}$; the combined set for cluster $j$, using the following fuzzy compositional rule of inference (CRI) [5] for $\mu_{H j}^{+}(h), \mu_{S j}^{+}(s), \mu_{I j}^{+}(i), \mu_{P j}(h, s, i) \in$ $[0.1] \forall h, s, i \in[0,255]$ as follows:

$$
\mu_{P j}(h, s, i)=\mu_{H j}^{+}(h) \wedge \mu_{S j}^{+}(s) \wedge \mu_{I j}^{+}(i),
$$

where $\mu_{X j}(x)$ is the membership function mapping values $x$ into membership values in set $X_{j}$ and $\mu_{p} \wedge \mu_{q}=\min \left(\mu_{p}, \mu_{q}\right)$. The fuzzification process for the negative cluster set is identical but uses the fuzzy sets $H_{j}^{-}, S_{j}^{-}, I_{j}^{-}$, and $N_{j}$.

The fuzzified images, $P$ and $N$, are then generated using fuzzy union over all $P_{j}$ and $N_{j}$ given by

$$
\mu_{F}(h, s, i)=\mu_{F 1}(h, s, i) \vee \mu_{F 2}(h, s, i) \ldots \vee \mu_{F k}(h, s, i),
$$

where $F$ can be $P$ or $N$ and $\mu_{p} \vee \mu_{q}=$ $\max \left(\mu_{p}, \mu_{q}\right)$.

Then the neighborhood similarity measure of each pixel in the negative image set is computed. The similarity distance among the pixels is defined by a contextual distance threshold value.

The membership value of each pixel in the negative set, $N$, is then modified to incorporate spatial neighborhood support. The greater the sum confidence of the pixels in a pixel's neighborhood, the greater the pixel's membership value, i.e.,

$$
\begin{aligned}
\text { strengthen } \mu_{N}(\underline{x}) & \text { if }\left|\left\{\underline{y} \mid \mathrm{d}(\underline{x}, \underline{y})<d_{T}\right\}\right| \geq N_{T} \\
\text { weaken } \mu_{N}(\underline{x}) & \text { if }\left|\left\{\underline{y} \mid \mathrm{d}(\underline{x}, \underline{y})<d_{T}\right\}\right|<N_{T} .
\end{aligned}
$$

\section{DEFUZZIFICATION}

In this phase, linguistic meaning is assigned to each pixel in the set using a fuzzy inference operation [7], [8]. The fuzzy inference operation between positive and neighborhood-supportednegative sets is defined by a fuzzy if - then rule, of the form:

IF $\mu_{P}(\underline{x})>\mu_{\mathrm{N}}(\underline{x})$ THEN $\underline{x}$ is a member of postive set

\section{APPLICATION AND RESULTS}

This method has been applied in the development of ARIES (Autonomous Robotic Inspection Experimental System), an autonomous robot designed to inspect DOE warehouse waste storage drums for rust. In this project, the ARIES vision system is used to acquire drum surface images under controlled conditions and subsequently perform visual inspection leading to the classification of the drum as "acceptable" or "suspect". The upper images in Figure 3 illustrate drums which have significant rust along with potentially misleading (i.e., having colors within the range of colors that rust possesses) radiation hazard stickers. In the training phase, 


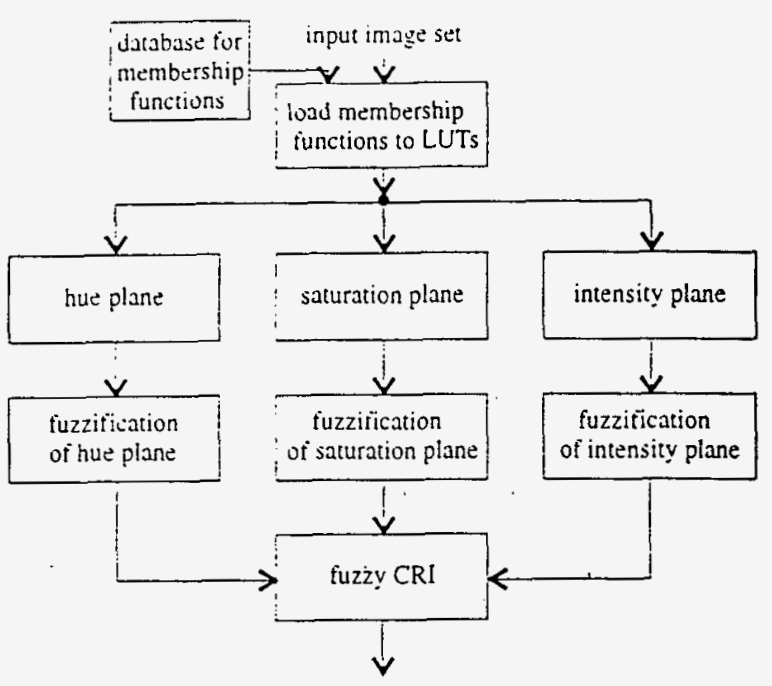

fuzzyfied image set

Fig. 2. Block diagram of fuzzification algorithm
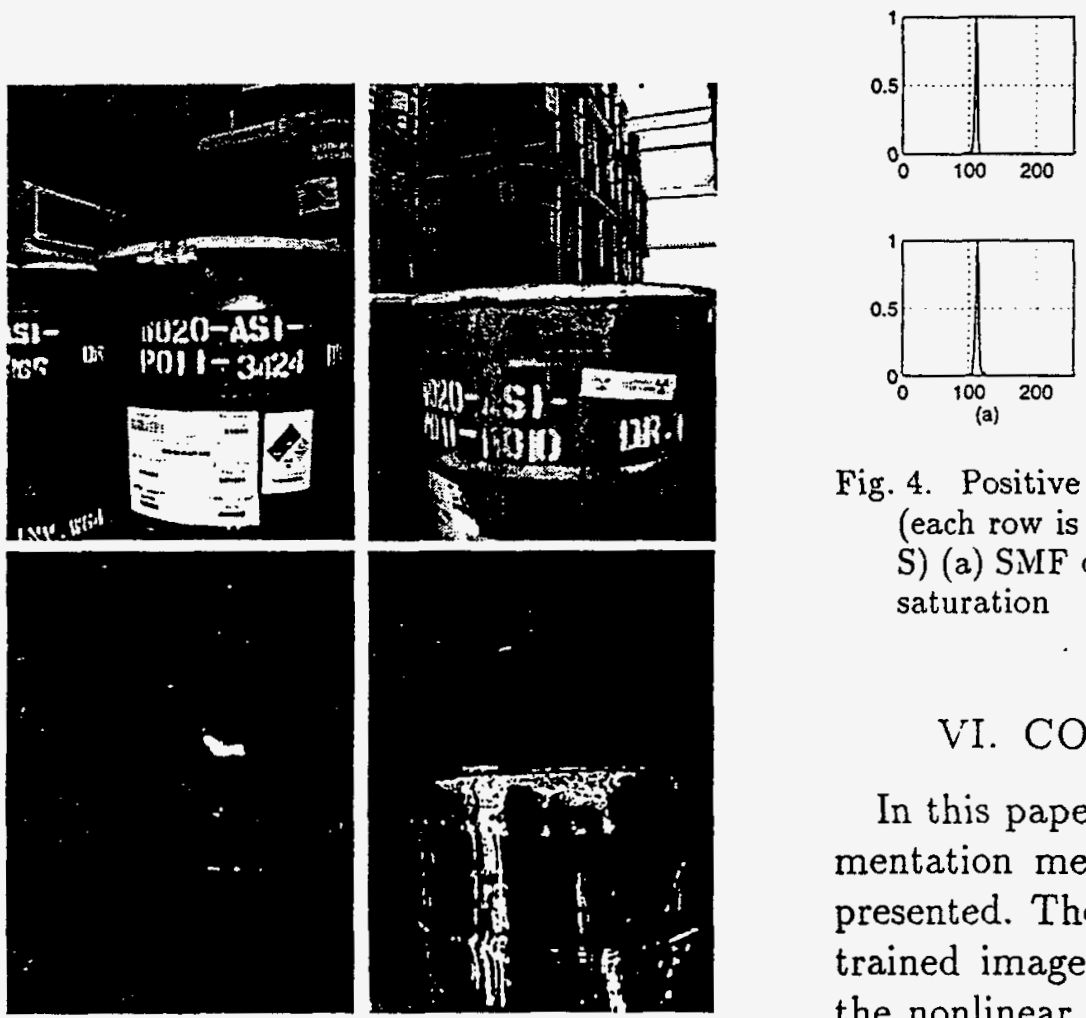

Fig. 3. Two color imagres anl segmented regions
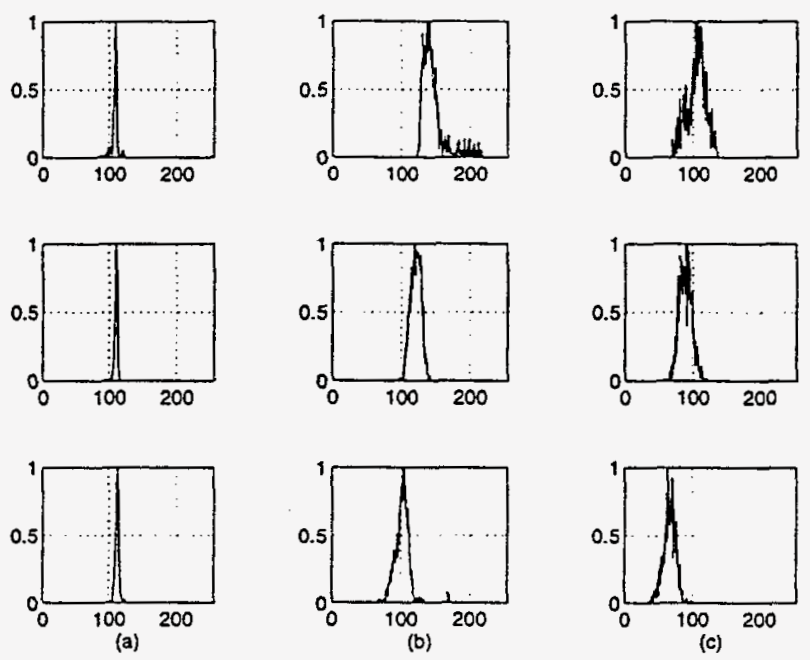

rust samples from four drum images were used to generate the positive membership functions. The false-positive misclassified pixels in these four images were then used to generate the negative membership functions. Figure 4 and 5 show the resultant positive and negative cluster similarity membership functions (SMF). These exemplars were then used to search for rust on 70 other images of drums with exceptional success. Greater than $95 \%$ of all rust pixels were found and all rust patches were indentified. Likewise, only a few pixels over all images were misclassified as rust, including those with potentially misleading stickers. Figure 3 shows two of these test input color images and the resulting segmented. regions.
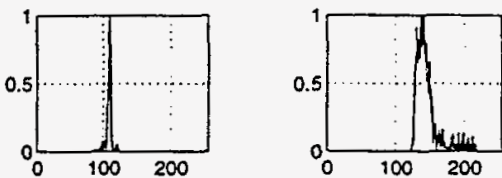

Fig. 4. Positive cluster similarity membership functions (each row is one cluster; columns represent $\mathrm{H}, \mathrm{I}$ and S) (a) SMIF of hue, (b) SiMF of intensity, (c) S.MF of saturation

\section{CONCLUDING REMARKS}

In this paper, a novel image analysis and segmentation method using fuzzy set concepts is presented. The learning phase is a generic, usertrained image analysis method which develops the nonlinear domain knowledge in the form of fuzzy membership functions which characterize positive and negative exemplars. The segmentation phase employs this knowledge using fuzzy 
set concepts to map an HSI image to a set of correpsonding confidence values for being members of the positive and negative sets. In the defuzzification phase, these two confidence value sets are combined to yield crisp classifications for all pixels as being either "positive" or "negative" pixels. The results from its application to a set of real images illustrates the quality and robustness of the concept.
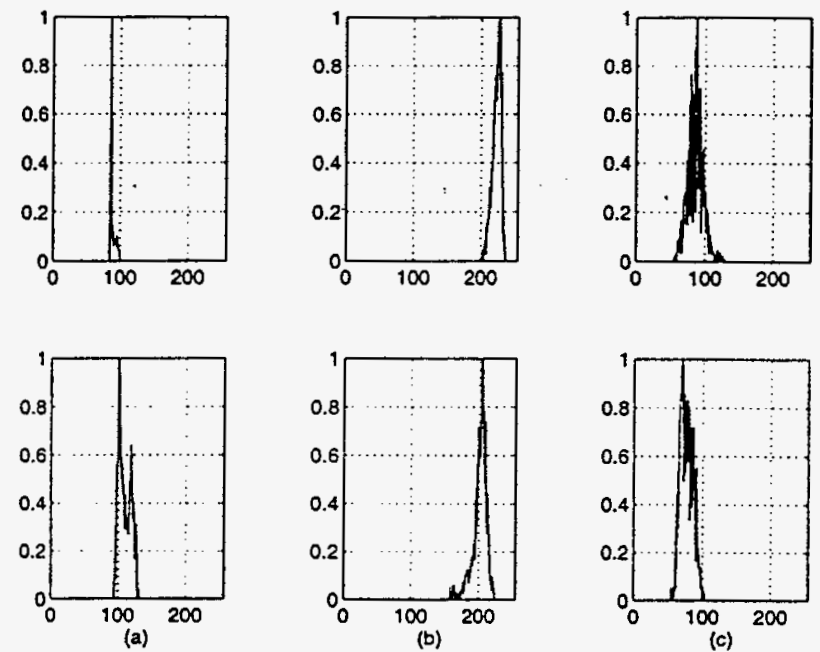

Fig. 5. Negative cluster similarity membership functions (each row is one cluster; columns represent $\mathrm{H}, \mathrm{I}$ and S) (a) SMF of hue, (b) SMF of intensity, (c) SMF of saturation

\section{REFERENCES}

[1] S. Lee B. Bhanu and S. Das. Adaptive image segmentation using genetic and hybrid search methods. IEEE Transec. tions on Aerospace and Electronic Systems, 31:1268-1290, 1995.

[2] K. R. Castleman. Digital Image Processing. Prentice-Hall, Inc., 1996.

[3] The ITI Group. ITI Manual. Imageing Technology Incorporated, 55 Middlesex Turnpike, Bedford, MA 01730 $1421,1994$.

[4] A. K. Jain. Fundamentals of Digital Image Processing. Prentice-Hall, Inc., 1989.

[5] A. Kaufmann. Introduction to the Theory of Fuzzy Subsets Volume 1. Academics Press, 1975.

[6] R. J. Schalkoff. Pattern Recognition: Statistical, Structural and Neural Approaches. John Wiley \& Sons., 1992.

[7] R. J. Schalkoff. Artificial Neural Networks. McGraw-Hill, 1997.

[8] H. Y. Hwang Y. S. Chen and B. T. Chen. Color image analysis using fuzzy set theory. IEEE, 1:242--245, 1995. 


\section{M98051220 \\ |||||||||||||||||||||||||||||||||||||||||||||||}

Report Number (14) DOE/EETC/C-
CONE-9817304

دubl. Date (11) $\quad 997 / 2$

sponsor Code (18) DOE/FE, XF

JC Category (19) $U C=1 O 1, D O E / E R$ 